J. Clin. Chem. Clin. Biochem.

Vol. 15, 1977, pp. 457-464

\title{
Vollenzymatische Triglycerid-Bestimmung: Präzision, Richtigkeit, Methodenvergleich
}

\author{
Von P. H. Müller, R.-M. Schmülling, H. M. Liebich, M. Eggstein
}

Medizinische Klinik, Abteilung Innere Medizin IV (Direktor: Prof. Dr. M. Eggstein) der Universität Tübingen,

F. Stähler und $K$. Stinshoff

Biochemica Werk Tutzing, Boehringer Mannheim $\mathrm{GmbH}$

(Eingegangen am 13. August 1976/5. Mai 1977)

Herrn Prof. Dr. Dr. h. c. G. Schettler zum 60. Geburtstag gewidmet

Zusammenfassung: Mit der von Eggstein \& Kreutz (Eggstein, M. \& Kreutz, F. H. (1966), Klin. Wochenschr. 44, 262-267; Eggstein, M. \& Kuhlmann, Elisabeth (1974) in Methoden der enzymatischen Analyse (Bergmeyer, H. U., ed.) 3. Aufl., S. 1871-1878, Verlag Chemie, Weinheim, Bergstr.) entwickelten enzymatischen Bestimmung der Triglyceride über Glycerin vor und nach alkalischer Verseifung wird eine vollenzymatische Triglyceridmessung verglichen, bei der die Verseifung mit Hilfe eines Lipase-Esterase-Gemisches erfolgt.

Die Praktikabilität dieser vollenzymatischen Methode übertrifft das bisherige Verfahren. Die enzymatische Verseifung erfolgt bei $37{ }^{\circ} \mathrm{C}$ oder Raumtemperatur. Die Serumproben können direkt zum Reaktionsgemisch gegeben und der Arbeitsgang auf drei Pipettierungen reduziert werden. Die analytische Präzision der vollenzymatischen Methode war im Meßbereich von 2 bis $7 \mathrm{mmol} / 1$ ( 177 bis $620 \mathrm{mg} / 100 \mathrm{ml})$ mit Variationskoeffizienten zwischen 0,5 und $4 \%$ gut. Im unteren Meßbereich bei einer Triglycerid-Konzentration von $0,7 \mathrm{mmol} / \mathrm{l}(62 \mathrm{mg} / 100 \mathrm{ml})$ Serum wurde ein mittlerer Variationskoeffizient bis zu 9\% errechnet, bei extrem hohen Triglycerid-Konzentrationen bis $70 \mathrm{mmol} / \mathrm{l}$ $(6195 \mathrm{mg} / 100 \mathrm{ml})$ Serum stieg der Variationskoeffizient auf 7\%. Die Originalmethode von Eggstein \& Kreutz ((1966), Klin. Wochenschr. 44, 262-267) ergab für den Bereich von 5-70 mmol/1 (443-6195 mg/100 ml) Serum Variationskoeffizienten unter 1,5\%. Die Richtigkeit der beiden Methoden wurde mit Glycerin- und Triolein-Lösungen und durch Aufsstockungsversuche belegt. Störende Einflüsse durch Hämolyse, Hyperbilirubinämie und durch 59 häufig verordnete Medikamente konnten nicht nachgewiesen werden. Die Korrelation der beiden Methoden ergab für die vollenzymatische Methode systematisch etwa 10\% niedrigere Ergebnisse als für die Originalmethode Eggstein \& Kreutz. Die Überprüfung des Gehaltes an freiem Glycerin im Serum bestätigte die Literaturangaben, daß für diagnostische RoutineTriglycerid-Bestimmungen ein Pauschalbetrag für das freie Glycerin in Abzug gebracht werden kann.

\section{A fully enzymatic triglyceride determination}

Summary: A fully enzymatic triglyceride determination utilizing enzymatic hydrolysis with a lipase-esterase-mixture is compared with the enżymatic determination by Eggstein \& Kreutz (Eggstein, M. \& Kreutz, F. H. (1966), Klin. Wochenschr. 44, 262-267; Eggstein, M. \& Kuhlmann, Elisabeth (1974) in Methoden der enzymatischen Analyse (Bergmeyer, H. U. ed.) 3. ed., pp. 1871-1878, Verlag Chemie, Weinheim, Bergstr.) which is based on the glycerol determination before and after alkaline saponification.

The fully enzymatic method is the more practicable. The enzymatic hydrolysis is performed at $37^{\circ} \mathrm{C}$ or at room temperature. The serum samples are added directly to the reaction mixture and the working process is reduced to three pipetting steps. With a relative standard deviation of 0.5 to $4 \%$ in the measuring range of 2 to $7 \mathrm{mmol} / \mathrm{l}$ $(177$ to $620 \mathrm{mg} / 100 \mathrm{ml}$ ) the analytical precision was good. In the lower measuring range with a concentration of triglycerides in serum of $0.7 \mathrm{mmol} / 1(62 \mathrm{mg} / 100 \mathrm{ml})$ a mean relative standard deviation of up to $9 \%$ was calculated; for the extremely high concentrations of triglycerides up to $70 \mathrm{mmol} / 1(6195 \mathrm{mg} / 100 \mathrm{ml})$ a relative standard deviation of $7 \%$ was found. For the range of concentration of 5 to $70 \mathrm{mmol} / 1(443-6195 \mathrm{mg} / 100 \mathrm{ml})$ serum the original method by Eggstein \& Kreutz ((1966), Klin. Wochenschr. 44, 262-267) had a relative standard deviation of below $1.5 \%$. The accuracy of the two methods was established with solutions of glycerol and triolein and by standard addition experiments. No interference was observed from hemolysis, hyperbilirubinemia, or frequently 
administered drugs. The correlation of the two methods proved that the results from the fully enzymatic method were systematically lower by $10 \%$ than the results from the method by Eggstein \& Kreutz. The examination of the free glycerol in serum confirmed the findings reported in the literature, that for routine determinations of triglycerides and for diagnostic purposes the subtraction of a global amount for the free glycerol is satisfactory.

\section{Einleitung}

Neben der quantitativen Bestimmung des Serumcholesterins kommt wahrscheinlich auch den Triglyceriden eine Bedeutung als Risikoindikator für arteriosklerotische, koronare und periphere Gefäßerkrankungen zu (1). Beide Verfahren liefern die Kardinalsymptome zur Klassifizierung der primären Hyperlipoproteinämien. Sekundäre Blutfettverschiebungen, Resorptions- und Ernährungsstudien verlangen ebenfalls eine spezifische und präzise Messung der Triglyceride im Blut.

Das von Eggstein \& Kreutz 1966 (2) beschriebene Verfahren zur enzymatischen Neutralfettbestimmung besitzt den Rang einer „empfohlenen Methode“ (Selected Routine Methode, Büttner et al. (3)) mit entsprechender Verbreitung in der laboratoriumsdiagnostischen Analytik.

Aus Gründen der arbeitstechnischen Rationalisierung, der „Praktikabilität von Methoden“, muß der bislang manuell durchgeführte Schritt der chemischen Verseifung als verbesserungswürdig und - für die Anpassung der Methode an Analysenautomaten - als hinderlich angesehen werden. Chemische Lösungen dieses Problems (z. B. Soloni (4), Tiffany et al. (5), Giegel et al. (6), Neri \& Frings (7), Köhring \& Kattermann (8)) durch Übergang auf andere Extraktions- und Verseifungsverfahren vermochten sich nicht durchzusetzen.

Die Arbeiten von Bucolo \& David (9) und von Wahlefeld (10) führten zur Entwicklung eines Lipase. Esterase-Gemisches mit einer der chemischen Verseifung vergleichbaren lipolytischen Wirkung. Damit wird die vollenzymatische Triglycerid-Bestimmung in einer einzigen Arbeitslösung im Prinzip durchführbar.

Präzision, Richtigkeit und Vergleichbarkeit zur bisherigen Triglycerid-Bestimmung mit alkalischer Verseifung werden untersucht.

Die Erprobung nach Adaptation der Bestimmung an Endpunkt-Automaten wird zur Zeit durchgeführt. Wir verfügen über eine Weiterentwicklung des Vcrfahrens zur vollenzymatischen Triglycerid-Bestimmung in ,Continuous Flow Automaten" vom Typ Technicon Autoanalyzer der I. und II. Generation $(11,12)$.

\section{Methoden}

Die Triglyceride im Serum werden nach Hydrolyse der GlycerinFettsäure-Ester über die quantitative Bestimmung des Glycerins errechnet. Das zugrunde gelegte Molekulargewicht von 885 (13) entspricht Triolein. Es stimmt mit dem mittleren Molekulargewicht von Serumtriglycerid-Gemischen überein (14).
Der Gehalt des Serums an freiem, unverestertem Glycerin wird bei der Berechnung in Abzug gebracht. Aus einem doppelten Ansatz mit und ohne Triglycerid-Hydrolyse kann aus der Differenz des "Gesamt-Glycerins" und des ,freien Glycerins" das "Triglycerid-Glycerin" bestimmt und daraus - in der Annahme, daß im menschlichen Plasma Di- und Monoglyceride nur in Spuren vorkommen - die Konzentration der Triglyceride berechnet werden.

Da der Anteil des freien Glycerins am gesamten Glycerin nach Hydrolyse klein ist, begnügt man sich unter Routinebedingungen mit einer allgemeinen Korrektur und verzichtet auf die Bestimmung des freien Glycerins. Folgende Berechnungen der SerumTriglyceride sind üblich:

1. „unnkorrigierte Triglyceride" unter Vernachlässigung des freien Glycerins im Gesamt-Glycerin,

2. „,korrigierte Triglyceride "über ein allgemeines Korrekturglied für freies Glycerin,

3. "wahre Triglyceride" unter Berücksichtigung des freien Glycerins.

In dieser Arbeit wurde der Anteil des freien Glycerins nicht gesondert berücksichtigt; für den Methodenvergleich wurden ausschließlich „unkorrigierte Triglycerid-Werte" verwandt.

\section{Methodenprinzip Eggstein \& Kreutz (15)}

\section{Triglycerid-Hydrolyse}

Durch ethanolische Alkalilösung wird die Esterbindung der Triglyceride verseift. Es entstehen Glycerin und die Alkalisalze der freien Fettsäuren.

Triglyceride $\stackrel{\mathrm{KOH}, 70^{\circ} \mathrm{C}}{\longrightarrow}$ Glycerin + Fettsäuren

\section{Meßreaktion}

Die quantitative Bestimmung des Glycerins erfolgt in einem zusammengesetzten optischen Test:

\begin{tabular}{|c|c|c|c|}
\hline Glyceriñ & + ATP $\stackrel{\text { GK }}{\rightleftharpoons}$ & $G=3-P$ & $+\mathrm{ADP}$ \\
\hline ADP & $+\mathrm{PEP} \stackrel{\mathrm{PK}}{\rightleftharpoons}$ & ATP & + Pyruvat \\
\hline Pyruvat + & $\mathrm{NADH}+\mathrm{H}^{+}$ & Lactat & $+\mathrm{NAD}^{+}$ \\
\hline $\begin{array}{l}\text { GK: } \\
\text { G-3-P: } \\
\text { PEP: } \\
\text { PK: } \\
\text { LDH: }\end{array}$ & \multicolumn{3}{|l|}{$\begin{array}{l}\text { Glycerokinase } \\
\text { D-Glycerin-3-phosphat } \\
\text { Phosphoenolpyruvat } \\
\text { Pyruvat-Kinase } \\
\text { Lactat-Dehydrogenase }\end{array}$} \\
\hline
\end{tabular}

\section{Vollenzymatische Methode}

Triglycerid-Hydrolyse

Bei dieser Methode werden die Triglyceride durch ein LipaseEsterase-Gemisch (Carboxy-esterase) in Glycerin und Fettsäuren gespalten.

Triglyceride $\stackrel{\text { Lipase:-Esterase }}{\longrightarrow}$ Glycerin + Fettsäuren

\section{Meßreaktion}

Die Bestimmung des Glycerins wird nach der gleichen Meßreaktion wie bei der Methode Eggstein,\& Kreuiz durchgefuhrt. 


\section{Arbeitsgang}

\section{Originalmethode}

Bei der Originalmethode wird nach der Serum-Hydrolyse und der nachfolgenden Perchlorat-Neutralisation der Eiweiß-PerchloratNiederschlag abzentrifugiert. Vom (iberstand (Hydrolysat) wird eine Probe zum Reaktionsgemisch gegeben und die Meßreaktion mit Glycerokinase-Lösung gestartet.

\section{Vollenzymatische Methode}

Bei der enzymatischen Hydrolyse werden $0,05 \mathrm{ml}$ Serum zu $2,5 \mathrm{ml}$ Reaktionsgemisch gegeben und bei $37^{\circ} \mathrm{C}$ für 25 min inkubiert. Nach dem Testprinzip der Originalmethode wird anschließend die Meßreaktion gestartet. Die Neutralisation, Zentrifugation und das Überpipettieren in den Meßansatz entfällt.

\section{Methodenvergleich}

Der Vergleich der Methode Eggstein \& Kreutz und der vollenzymatischen Methode wurde nach 3 verschiedenen Verfahren durchgeführt.

\section{Verfahren I}

$0,05 \mathrm{ml}$ Serum in $2,5 \mathrm{ml}$ Reaktionsgemisch werden $25 \mathrm{~min}$ bei $37^{\circ} \mathrm{C}^{1}$ ) inkubiert, nach Zugabe von $0,01 \mathrm{ml}$ Glycerokinase-Lösung wird bei Raumtemperatur nach 12 min die Absorption der Probe $\left(A_{P}\right)$ und in einer Vergleichsküvette bei entsprechendem Ansatz die Absorption des Proben-Leerwerts $\left(A_{L}\right)$ mit Wasser anstelle von Glycerokinase-Lösung am Photometer abgelesen.

$$
\Delta A=A_{P}-A_{L}
$$

\section{Verfahren II}

$2,5 \mathrm{ml}$ Reaktionsgemisch und $0,05 \mathrm{ml}$ Serum werden $25 \mathrm{~min}$ bei $37^{\circ} \mathrm{C}^{1}$ ) inkubiert, dann Absorption $A_{1}$ gemessen. Start der Meßreaktion mit $0,01 \mathrm{ml}$ Glycerokinase-Lösung, nach $12 \mathrm{~min} \mathrm{Ab}$ sorption $\mathbf{A}_{2}$ messen.

$$
\Delta A=A_{1}-A_{2}
$$

\section{Verfahren III}

Bei dieser Bestimmung wird wie bei Verfahren II vorgegangen. Um mögliche Nebenreaktionen zu erfassen, wurde zusätzlich nach weiteren $12 \mathrm{~min}$ noch eine Absorption $\mathrm{A}_{3}$ abgelesen.

$$
\Delta A=\left(A_{1}-A_{2}\right)-\left(A_{2}-A_{3}\right)
$$

Bei jeder der drei Meßreihen wurde ein Reagenzien-Leerwert berücksichtigt. Es wurde nur frisches Serum verwandt. Lipämische Seren müssen vor Gebrauch gut durchmischt werden. Stark lipämische Seren wurden $1+9 \mathrm{mit} 9 \mathrm{~g} / \mathrm{l} \mathrm{NaCl}$-Lösung verdünnt.

\section{Materialien}

Die Materialien zur Durchführung der Originalmethode entsprechen den Angaben von Eggstein in Bergmeyer: ,Methoden der enzymatischen Analyse" (15). Die Materialien für die vollenzymatische Triglyçerid-Bestimmung sind:

\section{Reagenzien}

1. Lipase (EC 3.1.1.3), aus Rhizopus arrhizus

2. Esterase (EC 3.1.1.1), aus Schweineleber

3. Glycerokinase (EC 2.7.1.30) aus Candida mycoderma

4. Lactatdehydrogenase (EC 1.1.1.27) aus Schweineherz

5. Pyruvatkinase (EC 2.7.1.40) aus Kaninchenmuskel

6. di-Natriumhydrogenphosphat, $\mathrm{Na}_{2} \mathrm{HPO}_{4} \times 2 \mathrm{H}_{2} \mathrm{O}$, p. a.

7. Natrium-dị-hydrogenphosphat, $\mathrm{NaH}_{2} \mathrm{PO}_{4} \times \mathrm{H}_{2} \mathrm{O}$, p. a.

1) Mit einer Esterase mikrobiellen Ursprungs wird neuerdings die Triglycerid-Hydrolyse in kürzerer Zeit $(15 \mathrm{~min})$ und bei Raumtemperatur erreicht.
8. Magnesiumsulfat, $\mathrm{MgSO}_{4} \times 7 \mathrm{H}_{2} \mathrm{O}$, p. a.

9. NADH als Dinatriumsalz, $\beta$-NADH-Na

10. ATP als Dinatriumsalz, ATP- $-\mathrm{Na}_{2} \mathrm{H}_{2} \times 3 \mathrm{H}_{2} \mathrm{O}$

11. Phosphoenolpyruvat als Tricyclohexylammoniumsalz

12. Glycerin wasserfrei $D=1,26 \mathrm{~kg} / 1$ reinst

13. Triolein (Trioleylglycerin), Reinheit $99 \%$, Fa. Roth, Kairlsruhe

14. Ethanol abs., p. a.

Die Bestimmungsansätze ivurden mit Enzymen der Fa. Boehringer Mannheim durchgeführt.

\section{Konzentration der Lösungen}

Lösung 1: $50 \mathrm{mmol} / \mathrm{l}$ Phosphat-Puffer, $\mathrm{pH}=7,0$

$2 \mathrm{mmol} / \mathrm{l}$ Magnesiumsulfat

Lösung 2: $10 \mathrm{mmol} / 1 \mathrm{NADH}$

$25 \mathrm{mmol} / 1$ ATP

$19 \mathrm{mmol} / \mathrm{l}$ Phosphoenolpyruvat

Lösung 3: $300 \mathrm{kU} / 1$ Lactatdehydrogenase

$50 \mathrm{kU} / \mathrm{l}$ Pyruvatkinase

$5 \mathrm{MU} / 1$ Lipase

$400 \mathrm{kU} / 1$ Esterase

Lösung 4: $150 \mathrm{kU} / \mathrm{l}$ Glycerokinase

Das Reaktionsgemisch wurdc täglich frisch angesetzt (nach Bedarf ein Vielfaches der angegebenen Mengen).

Reaktionsgemisch: Lösung 1: $5,0 \mathrm{ml}$

Lösung 2: $0,1 \mathrm{ml}$

Lösung 3: $0,1 \mathrm{ml}$

\section{Geräte}

Photometer Eppendorf mit Thermostat, Wechselautomatik und Registriereinrichtung; Messung bei $334 \mathrm{~nm}$. Laborzentrifuge. Wasserbad. Normal- und Halbmikro-Glasküvetten.

\section{Untersuchungsgut}

Der Methodenvergleich wurde durchgeführt mit wäßrigen Glycerin- und ethanolischen Triolein-Standardlösungen und mit Patientenseren der Medizinischen Universitätsklinik und ihrer Fcttstoffwechselambulanz. Bei Zumisch-Versuchen erfolgte die Zugabe von Glycerin bzıv. Triolein in ethanolischer Lösung.

\section{Ergebnisse und Diskussion}

\section{Technische Eigenschaften}

\section{Stabilität der Reagenzien}

Die Stabilität der Arbeitslösung (Reaktionsgemisch mit Lipase, Esterase, Pyruvatkinase, Lactatdehydrogenase, ATP, Phosphoenolpyruvat, NADH) wurde bei den 3 Lagerungstemperaturen $+4{ }^{\circ} \mathrm{C}$, Raumtemperatur und $33^{\circ} \mathrm{C}$ geprüft. Die Stabilität wurde als gewährleistet betrachtet, wenn die Wiederfindung in einem fraktioniert eingefrorenen, gepoolten Humanserum mit einer TriglyceridKonzentration von $1,65 \mathrm{mmol} / \mathrm{l}(146 \mathrm{mg} / 100 \mathrm{ml})$ bei $100 \pm 5 \%$ des Sollwertes lag. Der Sollwert und Streubereich waren zu Beginn des Experimentes in einer 20fach-Analyse ermittelt worden.

Die Arbeitslösung ist bei $+4{ }^{\circ} \mathrm{C} 7$ Tage, bei Raumtemperatur 2 Tage und bei $33^{\circ} \mathrm{C}$ maximal 8 Stunden haltbar (Abb. 1).

Die zum Start der Reaktion eingesetzte GlycerokinaseSuspension ist bei allen Temperaturen mindestens 4 Wochen stabil. 


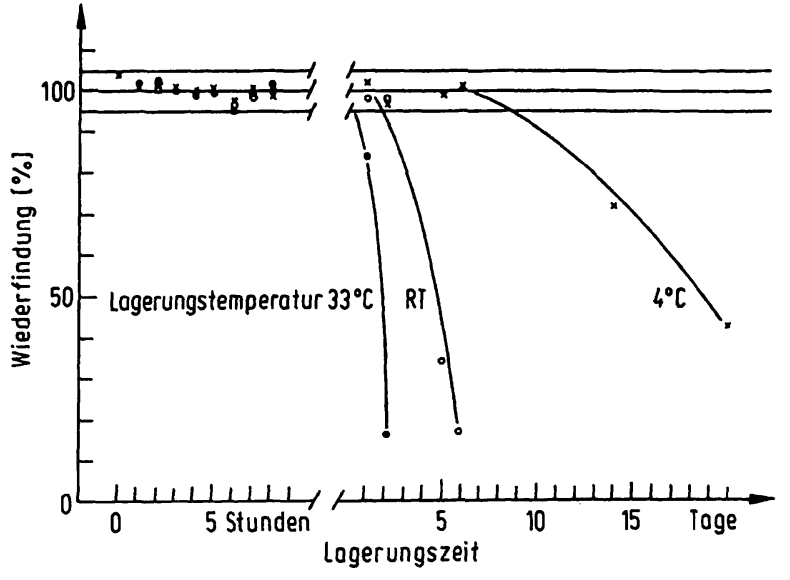

Abb. 1. Stabilität der Arbeitslösung für verschiedene Lagerungstemperaturen und Lagerungszeiten. $\mathrm{RT}=$ Raumtemperatur.

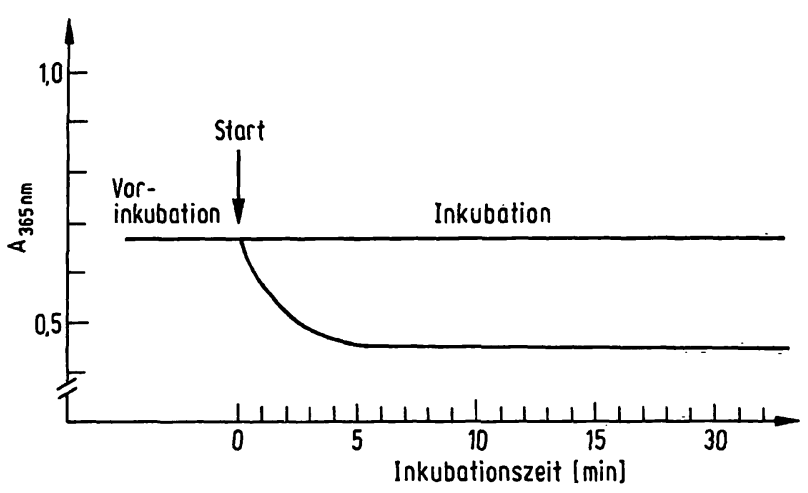

Abb. 2. Reaktionsverlauf: $10 \mathrm{~min}$. Vorinkubation von $0,1 \mathrm{ml}$ Serum (Triglycerid-Gehalt: $5,16 \mathrm{mmol} / 1=457 \mathrm{mg} / 100 \mathrm{ml}$ ) mit 5,0 ml Arbeitslösung, dann Teilung des Ansatzes und Start der Reaktion mit 0,01 ml Glycerokinase-Lösung.

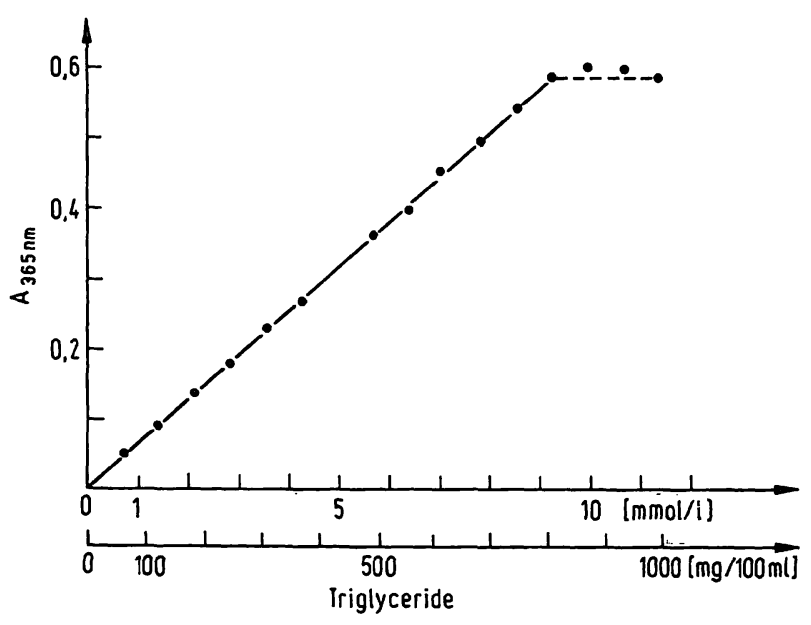

Abb. 3. Abhängigkeit des Meßsignals (A $365 \mathrm{~nm}$ ) von der Triglycerid-Konzentration (Verdünnungsreihe eines Humanserums mit $9 \mathrm{~g} / \mathrm{l} \mathrm{NaCl}$-Lösung), für $\mathrm{x} \leqslant 9,0 \mathrm{mmol} / \mathrm{l}$, $y=0,0635 x-0,0002, r=0,9998$

\section{Reaktionsverlauf}

Die Reaktion ist auch bei Vorlage höherer TriglyceridKonzentrationen nach $10 \mathrm{~min}$ abgelaufen (Abb. 2). Von der 10. bis. zur 30. min ist die Absorptionsdifferenz bis auf einen geringen Schleich von $A_{334} \mathrm{~nm}=0,002-$
$0,012 / 10 \mathrm{~min}$ (entsprechend einem Triglycerid-Gehalt von $0,02-0,09 \mathrm{mmol} / \mathrm{l} \mathrm{bzw.} 2-8 \mathrm{mg} / 100 \mathrm{ml}$ ) stabil. Bei unseren Untersuchungen wurde bei der Erweiterung des Ablesezeitraums auf 30 min maximal ein Fehler von 5\% durch unspezifischen NADH-Umsatz gefunden.

\section{Meßbereich und Linearität}

Die Linearität zwischen Triglycerid-Konzentration und Meßsignnal und auch der Meßbereich wurden durch stufenweise Verdünnung von 5 klaren und trüben Humanseren mit mäßig und stark erhöhtem Trïglycerid-Gehalt überprüft. Bis zu einer Konzentration von $7,90 \mathrm{mmol} / \mathrm{l}$ $(699 \mathrm{mg} / 100 \mathrm{ml})$ wurde eine lineare Absorptions-Konzentrationsbeziehung gefunden (Abb. 3). Unter Einbehaltung einer Sicherheitsreserve empfiehlt sich eine Begrenzung des Meßbereiches auf $6,80 \mathrm{mmol} / 1(602 \mathrm{mg} / 100 \mathrm{ml})$.

\section{Analy tische Eigenschaften}

\section{Präżision}

Die Präzision in der unterbrochenen Serie wurde in 5 - Konzentrationsbereichen mit der Originalmethode und den 3 Verfahren der vollenzymatischen Methode ermittelt (16). Die Ergebnisse sind in Tabelle 1 zusammengefaßt. Zur Beurteilung wurden die Methoden vom kleinsten zum größten Variationskoeffizienten rangiert. Die Originalmethode wurde mit der niedrigsten Rangsumme als beste Methode bewertet. Von den vollenzymatischen Verfahren hat Verfahren II mit der nächst niedrigsten Rangsumme das beste Ergebnis.

Entsprechend werden im Folgenden die Ergebnisse der Präzision in der Serie und von Tag zu Tag bewertet. Da von den vollenzymatischen Verfahren I, II und III immer Verfahren II die relativ beste Bewertung erhielt, beschränken wir uns auf den Vergleich der Originalmethode mit Verfahren II.

Die Präzision in der Serie und von Tag zu Tag wurte folgendermaßen ermittelt: In 10 fraktioniert eingefrorenen, gepoolten Humanserumproben mit verschiedenen Triglycerid-Konzentrationen wurden an 5 Tagen jeweils 5 fachBestimmungen durchgeführt. Die Reihenfolge der Analysen wurde jeden Tağ durch Randomisierung neu festgelegt. Pro Bereich ließen sich für jeden Tag eine Präzision in der Serie und aus der Reihe der jeweils ersten, zweiten, dritten, vierten und fünften Bestimmungen an jedem Tag eine Präzision von Tag zu Tag berechnen. Auf diese Weise ergaben sich für jeden der 10 Konzentrationsbereiche 5 Präzisionen in der Serie und je 5 Präzisionen von Tag zu Tag.

Die Ergebnisse sind in den Abbildungen 4a-c zusammengestellt. In Abbildung 4a ist die Präzision für Proben mit Konzentrationen innerhalb der Meßbereichsgrenzen dargestellt, sie zeigt eine Abhängigkeit vom Triglycerid-Gehalt. Im Entscheidungsbereich und darüber wurden mittlere Variationskoeffizienten bis $9 \%$ gefunden. In den Abbildungen $4 \mathrm{~b}+\mathrm{c}$ finden sịch die Daten für Seren mit 
Tab. 1. Präzision in der unterbrochenen Serie zur Bewertung der Methoden und der Verfahren I, II und III.

\begin{tabular}{|c|c|c|c|c|c|c|c|}
\hline & $\mathbf{N}$ & $(\mathrm{mmol} / \mathrm{l})$ & $(\mathrm{mg} / 100 \mathrm{ml})$ & $\begin{array}{l}\text { VK } \\
(\%)\end{array}$ & Rang & $\begin{array}{l}\text { Rang- } \\
\text { Summe }\end{array}$ & $\begin{array}{l}\text { Methoden- } \\
\text { Bewertung }\end{array}$ \\
\hline $\begin{array}{l}\text { Originalmethode } \\
\text { Eggstein \& Kreutz }\end{array}$ & $\begin{array}{l}25 \\
25 \\
25 \\
25 \\
15\end{array}$ & $\begin{array}{r}5,45 \\
5,64 \\
19,27 \\
28,06 \\
70,08\end{array}$ & $\begin{array}{r}482 \\
499 \\
1705 \\
2483 \\
6202\end{array}$ & $\begin{array}{l}0,9 \\
0,6 \\
0,3 \\
1,9 \\
1.0\end{array}$ & $\begin{array}{l}1 \\
1 \\
1 \\
2 \\
1\end{array}$ & 6 & 1 \\
\hline $\begin{array}{l}\text { Verfahren } I \\
\Delta A=A_{P}-A_{L}\end{array}$ & $\begin{array}{l}25 \\
25 \\
25 \\
25 \\
15\end{array}$ & $\begin{array}{r}4,95 \\
5,02 \\
17,11 \\
25,33 \\
70.99\end{array}$ & $\begin{array}{r}438 \\
444 \\
1514 \\
2242 \\
6283\end{array}$ & $\begin{array}{l}3,6 \\
2,0 \\
1,8 \\
3.2 \\
7,0\end{array}$ & $\begin{array}{l}3 \\
2 \\
4 \\
4 \\
4\end{array}$ & 17 & 4 \\
\hline $\begin{array}{l}\text { Verfahren II } \\
\Delta A=A_{1}-A_{2}\end{array}$ & $\begin{array}{l}25 \\
25 \\
25 \\
25 \\
15\end{array}$ & $\begin{array}{r}4,89 \\
5,05 \\
17,31 \\
23,93 \\
70.17\end{array}$ & $\begin{array}{r}433 \\
447 \\
1532 \\
2118 \\
6210\end{array}$ & $\begin{array}{l}3,3 \\
2,6 \\
1,2 \\
1,6 \\
5,6\end{array}$ & $\begin{array}{l}2 \\
4 \\
2 \\
1 \\
2\end{array}$ & 11 & 2 \\
\hline $\begin{array}{l}\text { Verfahren III } \\
\begin{aligned} \Delta A= & \left(A_{1}-A_{2}\right) \\
& -\left(A_{2}-A_{3}\right)\end{aligned}\end{array}$ & $\begin{array}{l}25 \\
25 \\
25 \\
25 \\
15\end{array}$ & $\begin{array}{r}4.87 \\
5,02 \\
17,25 \\
25,15 \\
69,74\end{array}$ & $\begin{array}{r}431 \\
444 \\
1527 \\
2226 \\
6172\end{array}$ & $\begin{array}{l}3,6 \\
2,5 \\
1,5 \\
2,9 \\
5,8\end{array}$ & $\begin{array}{l}4 \\
3 \\
3 \\
3 \\
3\end{array}$ & 16 & 3 \\
\hline
\end{tabular}

Abb. 4. Mittlerer Variationskoeffizient (VK) in Abhängigkeit von der Triglycerid-Konzentration.

Leere Symbole: Einzel-VK für jede Serien-Reihe (Dreiecke) bzw. jede von-Tag-zu-Tag-Reihe (Kreise).

Volle Symbole: Arithmetisches Mittel der Einzel-VK in der Serie (Dreiecke) bzw. von Tag zu Tag (Kreise) für jeden Konzentrationsbereich.

Durchgezogene Linie (-): in der Serie

Unterbrochene Linie (...... - ) von Tag zu Tag

$\mathbf{x}$ : Triglycerid-Konzentration des Serumpools ( $\mathrm{mmol} / \mathrm{l}$ )

bzw. (mg/100 ml)

y: Variationskoeffizient (\%)

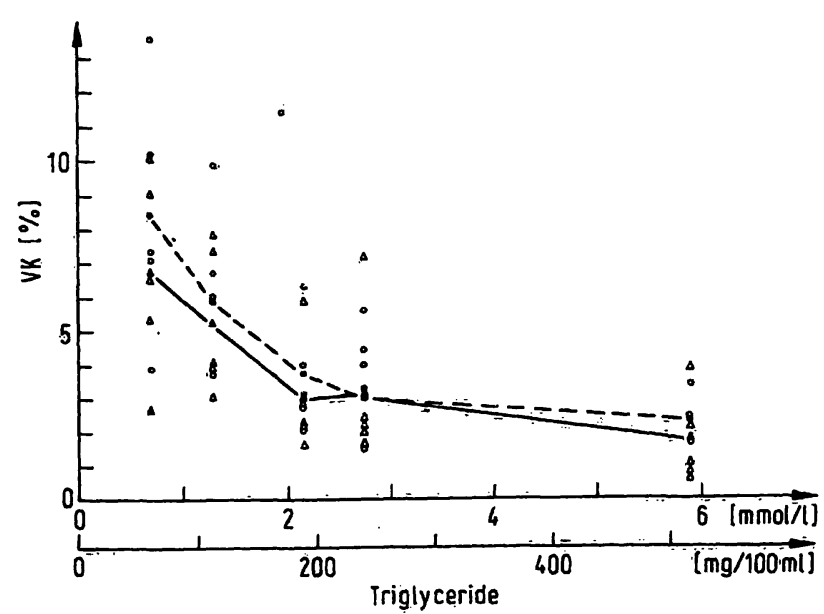

Abb. 4a. Präzision der vollenzymatischen Methode, Konzentrationsberejche unterhalb der Verdünnungsgrenze.

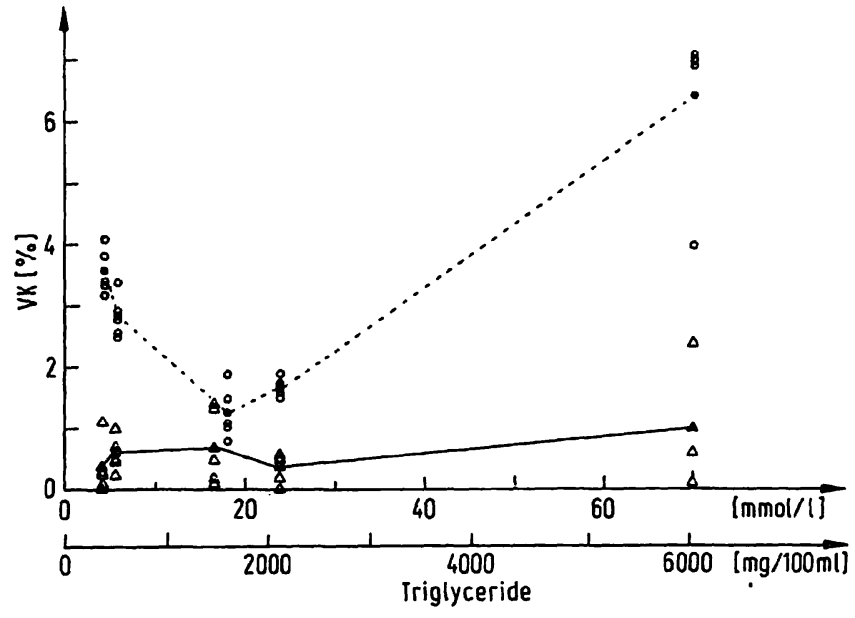

Abb. 4 b: Präzision der vollenzymatischen Methode, hohe Konzentrationsbereiche.

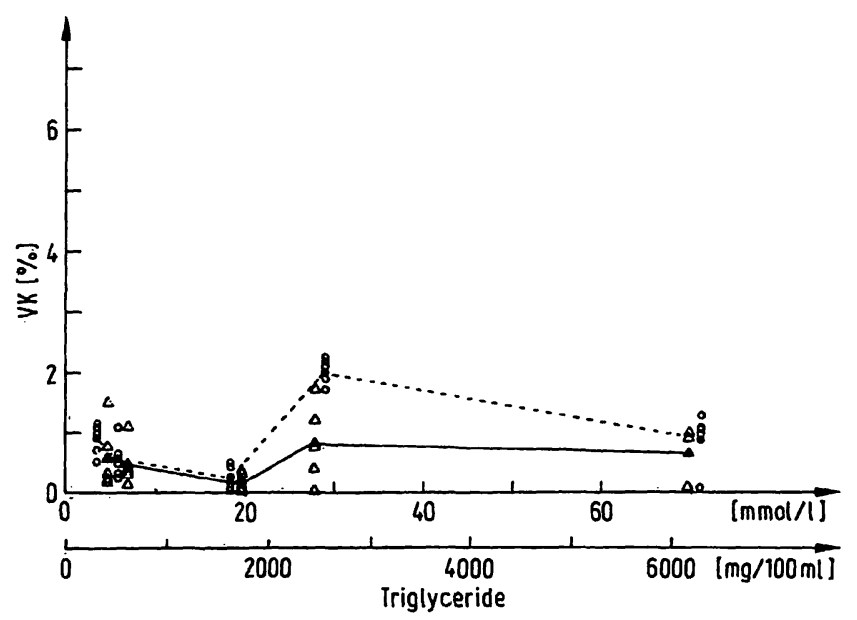

Abb. 4 c. Präzision der Methode Eggstein \& Kreutz, hohe Konzentrationsbereiche. 
stark erhöhtem Triglycerid-Gehalt (bis $68 \mathrm{mmol} / \mathrm{l} \mathrm{bzw}$. $6018 \mathrm{mg} / 100 \mathrm{ml}$ ). Aus ihnen folgt, daß in diesem Bereich die Präzision von Tag zu Tag der vollenzymatischen Methode im Vergleich zur Eggstein \& Kreutz-Methode ungünstiger ist (F-Test: $p \leqslant 0,001$, Wilcoxon-Test: $p \leqslant 0,01$ ).

\section{Richtigkeit}

\section{Wiederfindung von Glycerin}

Die Wiederfindung von Glycerin in wäßriger Lösung wurde weder durch den vorausgegangenen „Verseifungsund Neutralisationsschritt" noch durch das Enzymgemisch beeinflußt. Die Ausbeute stimmte im Rahmen der analytischen Präzision überein (Tab. 2).

\section{Wiederfindung von Triolein}

Die Vollständigkeit der Verseifung wurde an 5 Patientenseren, denen 3 verschieden konzentrierte ethanolische Triolein-Lösungen zugemischt wurden, geprüft (Tab. 3). Mit der enzymatischen Methode betrug die Wiederauffindung $\bar{x} \pm s=99,9 \pm 0,9 \%$. Die Sollwert-Wiederfindung bei Kalilauge-Verseifung betrug im Mittel 100,5 $\pm 1,1 \%$. Die Vollständigkeit der Triolein-Spaltung durch das eingesetzte Lipase-Esterase-Gemisch ist demzufolge auch gewährleistet.

Tab. 2. Wiederfindung von Glycerin in wäßriger Lösung Glycerin-Konzentration (Einwaage): $1,17 \mathrm{mmol} / 1$; $\mathrm{N}=20$.

\begin{tabular}{lllll}
\hline Methode & $\begin{array}{l}\bar{x} \\
(\mathrm{mmol} / \mathrm{l})\end{array}$ & $\begin{array}{l}\text { Abwei- } \\
\text { chung vom } \\
\text { Sollwert } \\
(\%)\end{array}$ & $\begin{array}{l}\mathrm{s} \\
(\mathrm{mmol} / \mathrm{l})\end{array}$ & $\begin{array}{l}\text { VK } \\
(\%)\end{array}$ \\
\hline $\begin{array}{l}\text { KOH-Verseifung } \\
\text { Enzymatische }\end{array}$ & 1,18 & $+1,2$ & 0,02 & 1,74 \\
$\begin{array}{l}\text { Verseifung } \\
\mathrm{A}_{1} / \mathrm{A}_{2} \text {-Ablesung }\end{array}$ & 1,17 & $+0,4$ & 0,05 & 4,05 \\
\hline
\end{tabular}

\section{Kontrollseren}

Im Vergleich zur Verseifung mit ethanolischer Kalilauge wurden nach enzymatischer Esterspaltung in Humanserum-Lyophilisaten (Kontrollseren ${ }^{2}$ ) im Mittel um 5-10\% niedrigere Werte (siehe auch unter Methodenvergleich an Patientenseren) gefunden. Die Ursache der Differenz konnte bisher experimentell nicht geklärt werden. Längere Inkubationszeiten und Erhöhung des Enzymgehaltes führten nicht zu einer Beseitigung der Differenz.

\section{Störungen}

1. Hämolyse und Hyperbilirubinämie

Hämoglobin bis zu einer Konzentration von $10 \mathrm{~g} / 1$ $(1 \mathrm{~g} / 100 \mathrm{ml})$ und Bilirubin bis $0,171 \mathrm{mmol} / 1$ $(10 \mathrm{mg} / 100 \mathrm{ml})$ stören den Test nicht.

\section{Pharmaka und Antikoagulantien}

Proben eines Humanserum-Pools wurden mit einer Auswahl von Medikamenten und Antikoagulantien (Tab. 4) versetzt. Die Konzentration der Pharmaka entsprach dabei der nach Gabe therapeutischer Maximaldosen. Eine Störung konnte auch in dieser Untersuchung nicht festgestellt werden, da die Wiederauffindung in keinem Fall außerhalb eines Bereiches lag, der durch den Mittelwert \pm doppelte Standardabweichung einer 10fach-Bestimmung der medikamentenfreien Kontrollprobe des gleichen Serumpools definiert war.

2) Hyland Europa-Kontrolle II

Monitrol I, Monitrol II

Precilip (Chargen: 323 A, 3.28 B, 429 B, 430 A. 433 A.

Validate A, Validate $\mathrm{N}$.

Tab. 3. Zumischversuche mit ethanolischen Triolein-Lösungen zu Patientenseren.

\begin{tabular}{lllcc}
\hline $\begin{array}{l}\text { Basiswert } \\
(\mathrm{mmol} / \mathrm{l})\end{array}$ & $\begin{array}{l}\text { Zumischung } \\
(\mathrm{mmol} / \mathrm{l})\end{array}$ & $\begin{array}{l}\text { Sollwert } \\
(\mathrm{mmol} / \mathrm{l})\end{array}$ & Verseifung mit $\mathrm{KOH}$ & $\begin{array}{c}\text { Ist-Werte } \\
\text { enzymatische Verseifung } \\
\text { (\% des Sollivertes) }\end{array}$ \\
\hline 1,26 & 1,35 & 2,61 & 100,4 & 100.9 \\
2,35 & 1,35 & 3,70 & 101,2 & 100 \\
2,25 & 1,35 & 3,60 & 100,9 & 100.3 \\
3,49 & 1,35 & 4,84 & 100,9 & 100,2 \\
3,78 & 1,35 & 7,13 & 100,8 & 100,8 \\
1,26 & 2,31 & 3,57 & 101,9 & 99,7 \\
2,35 & 2,31 & 4,66 & 100,9 & 100,2 \\
2,25 & 2,31 & 4,56 & 101,7 & 99,8 \\
3,49 & 2,31 & 5,80 & 100 & 100,6 \\
5,78 & 2,31 & 8,09 & 97,2 & 99 \\
1,26 & 3,45 & $4,71 \cdots$ & 100,5 & 100 \\
2,35 & 3,45 & 5,80 & 99,4 & 100 \\
2,25 & 3,45 & 5,70 & 100,4 & 99,8 \\
3,49 & 3,45 & 6,94 & 100,3 & 99,7 \\
5,78 & 3,45 & 9,23 & 100,2 & 97,2 \\
& & $\bar{x}$ & 100,5 & 99.9 \\
& & 5 & 1,1 & 0.9 \\
\hline
\end{tabular}


Tab. 4. Medikamente, Röntgen-Kontrastmittel und Antikoagulantien, deren Anwesenheit im Bestimmungsansatz keine nachweisbaren Störungen zeigte.

\begin{tabular}{|c|c|}
\hline Actol & - Acidum nifluminicum \\
\hline Aldactone & - Spironolactonum \\
\hline Amuno & - Indometacinum \\
\hline Angiografin & - Amidotrizoate sodium \\
\hline Antistin & $\begin{array}{l}\text { - Antazolinsulfat, Naphazolinnitrat, } \\
\text { Acid. boric. }\end{array}$ \\
\hline Aponal & - Doxepinum \\
\hline Aspirin & - Acidum acetosalicylicum \\
\hline Benemid & - Probenecidum \\
\hline Biligrafin & - Adipiodonum, Megluminum \\
\hline $\begin{array}{l}\text { Binotal } 500 \\
\text { Busconan }\end{array}$ & - Ampicillinum \\
\hline Buscopan & - Butylscopolaminum \\
\hline Butazolidin & - Phenylbutazonum \\
\hline Cebion & - Acidum ascorbicum \\
\hline Dipar & - Phenforminum \\
\hline Dolviran & $\begin{array}{l}\text { Acidum acetosalicylicum, Phenacetin, } \\
\text { Codeinum phosphoric., } \\
\text { Coffeinum anhydric., Phenobarbitalum }\end{array}$ \\
\hline Dulcolax & - Bisacodylum \\
\hline Dura Clofibrat & - Clofibratum \\
\hline Durenat & - Sulfamethoxydiazinum \\
\hline Endoxan & - Cyclophosphamidum \\
\hline Euglucon & - Glibenclamidum \\
\hline Furadantin & - Nitrofurantoinum \\
\hline Glifanan & - Glafeninum \\
\hline Hostacyclin & - Tetracyclinum \\
\hline $\begin{array}{l}\text { Hygroton } \\
\text { Intensain }\end{array}$ & - Chlorthalidonum \\
\hline $\begin{array}{l}\text { Intensain } \\
\text { Lanicor }\end{array}$ & - Carbocromenum \\
\hline $\begin{array}{l}\text { Lanicor } \\
\text { Lasix }\end{array}$ & $\begin{array}{l}\text { - Digoxinum } \\
\text { - Furosemidum }\end{array}$ \\
\hline $\begin{array}{l}\text { Lasix } \\
\text { Librium } 5\end{array}$ & $\begin{array}{l}\text { - Furosemidum } \\
\text { - Chlordiazepoxidum }\end{array}$ \\
\hline $\begin{array}{l}\text { Librium } 5 \\
\text { Luminal }\end{array}$ & - Phenobarbitalum \\
\hline Macrodex $6 \%$ & - Dextran \\
\hline Marcumar & - Phenprocoumonum \\
\hline Megaphen & - Chlorpromazinum \\
\hline Metalcaptase & - Penicillaminum \\
\hline Methotrexat & - Methotrexatum \\
\hline Miroton & $\begin{array}{l}\text { Standard. Pflanzenauszïge mit den } \\
\text { Gesamtglycosiden aus Extr. Bulb. } \\
\text { Scill. var. alb., Extr. Fol. Convall., } \\
\text { Extr. Fol. Oleandr., Extr. Herb. Adon. }\end{array}$ \\
\hline Modenol & $\begin{array}{l}\text { - Thiabutazid, Reserpin, Rescinnamin, } \\
\text { Raubasin, Kaliumchlorid }\end{array}$ \\
\hline Neoplasmagel $20 \%$ & $\begin{array}{l}\text { modifizierte, flüssige Gelatine, } \\
\text { Natriumchlorid, Calciumchlorid, } \\
\text { Elektrolyte }\end{array}$ \\
\hline Nicobion & - Nicotinsäureamid \\
\hline Novadral & - Norfenefrin- $\mathrm{HCl}$ \\
\hline Novalgin & $\begin{array}{l}\text { - Noramidopyrini methansulfonas } \\
\text { natricum }\end{array}$ \\
\hline Paraxin & - Chloramphenicolum \\
\hline Polybion & $\begin{array}{l}\text { Aneurinnitrat, Riboflavin, Nicotin- } \\
\text { säureamid, Calcium- } D \text {-pantothenat, } \\
\text { Pyridoxinhyydrochlorid, Cyanocobal- } \\
\text { amin, Biotin }\end{array}$ \\
\hline $\begin{array}{l}\text { Prolixan } \\
\text { Rastinon }\end{array}$ & $\begin{array}{l}\text { - Azapropapon } \\
\text { - Tolbutamidum }\end{array}$ \\
\hline Refobacin & - Gentamycinum \\
\hline Resoçhin & - Chloroquinum \\
\hline Sembrina & - Methyldopum \\
\hline Solu-Decortin & - Prednisolonum succinat natrium \\
\hline Sulfa-Furadantin & - Nitrofurantoin, Sulfadiazin \\
\hline Tanderil & - Oxyphenbutazonum \\
\hline Tolbutamid & - Tolbutamidum \\
\hline $\begin{array}{l}\text { Uricovac } \\
\text { Urografin }\end{array}$ & - Benzbromaronum \\
\hline $\begin{array}{l}\text { Urogiafin } \\
\text { Zyloric }\end{array}$ & $\begin{array}{l}\text { - Acidum amidotrizoicum } \\
\text { - Allopurinolumm }\end{array}$ \\
\hline $\begin{array}{l}\text { Na-Heparin } \\
\text { NaF } \\
\text { Na-Oxalat } \\
\text { Titriplex III/EDTA } \\
\text { Na-Citrat }\end{array}$ & \\
\hline
\end{tabular}

\section{Methodenvergleich}

Die Originalmethode von Eggstein \& Kreutz und die vollenzymatische Methode wurden an einem Kollektiv von 107 Seren von Patienten mit erhöhten Lipid-Spiegeln verglichen. Abbildung 5 zeigt das Ergebnis der Korrelationsanalyse. Die Regressionsgleichung lautet:

für $\mathrm{mmol} / 1$

$$
y=0,922 x-0,107, r=0,998-0,005
$$

für $\mathrm{mg} / 100 \mathrm{ml}$

$$
y=0,922 x-9,4, \quad r=0,998-0,005
$$

Der Korrelationskoeffizient $r \pm s_{r}$ von $0,998-0,005$ spricht für die Vergleichbarkeit beider Methoden. Das vollenzymatische Verfahren liefert Resultate, die im Mittel um $8 \%$ und zusätzlich $0,107 \mathrm{mmol} / 1$

$(9,4 \mathrm{mg} / 100 \mathrm{ml})$ niedriger liegen als die nach ethanolischer Kalilauge-Verseifung und unter Berücksichtigung des Leerwerts gemessenen Werte. Die bei den Kontrollseren beschriebene methodische Differenz von 5 bis $10 \%$ entspricht dieser Beobachtung.

\section{Einfluß des freien Glycerins}

Freies Glycerin und Gesamt-Glycerin wurden nach der vollenzymatischen Methode in 142 frischen Humanseren, unter denen der Anteil mit erhöhten Lipidwerten deutlich überrepräsentiert war, bestimmt. Aus diesen Daten wurden die jeweiligen wahren Triglycerid-Konzentrationen berechnet und mit unkorrigierten Triglyceriden korreliert (Abb. 6).

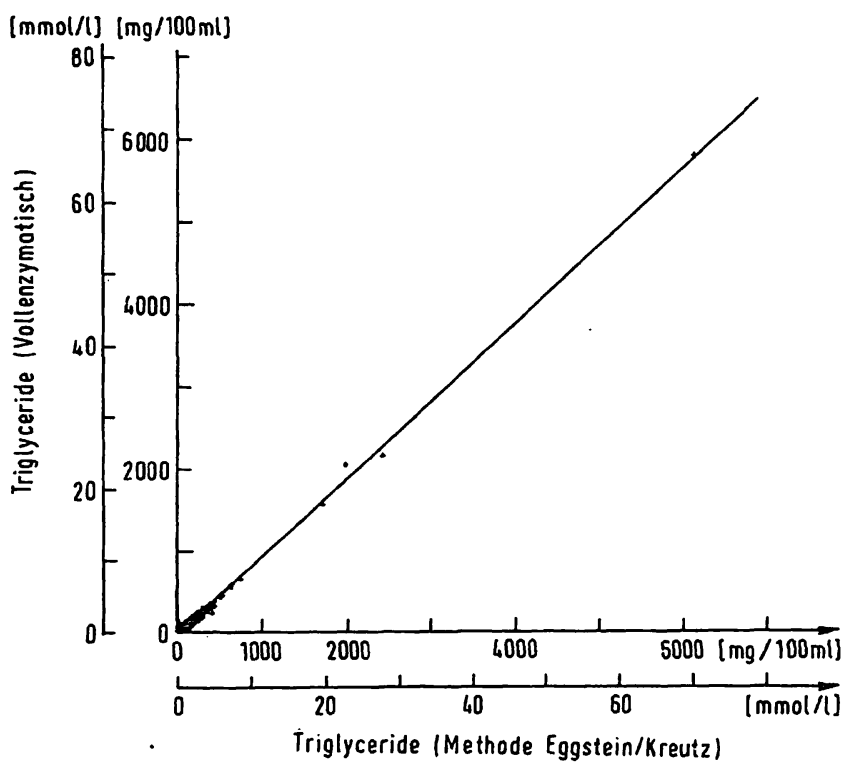

Abb. 5. Korrelation der Methode nach Eggstein \& Kreutz mit der vollenzymatischen Methode $\mathrm{N}=107$

$y=a+b x$

$a=-0,107($ für $\mathrm{mmol} / \mathrm{l}) \mathrm{a}=-9,4($ für $\mathrm{mg} / 100 \mathrm{ml}$ )

$\mathrm{b}=0,922 \pm 0,004$

$r=0,998 \pm 0,005$

$\overline{\mathrm{x}}=3,41 \pm 7,47$ (für mmol/l) $\overline{\mathrm{x}}=302 \pm 661$ (für $\mathrm{mg} / 100 \mathrm{ml}$ )

$\bar{y}=3,04 \pm 6,90$ (für mmol/l) $\bar{y}=269 \pm 611$ (für $\mathrm{mg} / 100 \mathrm{ml}$ ) 


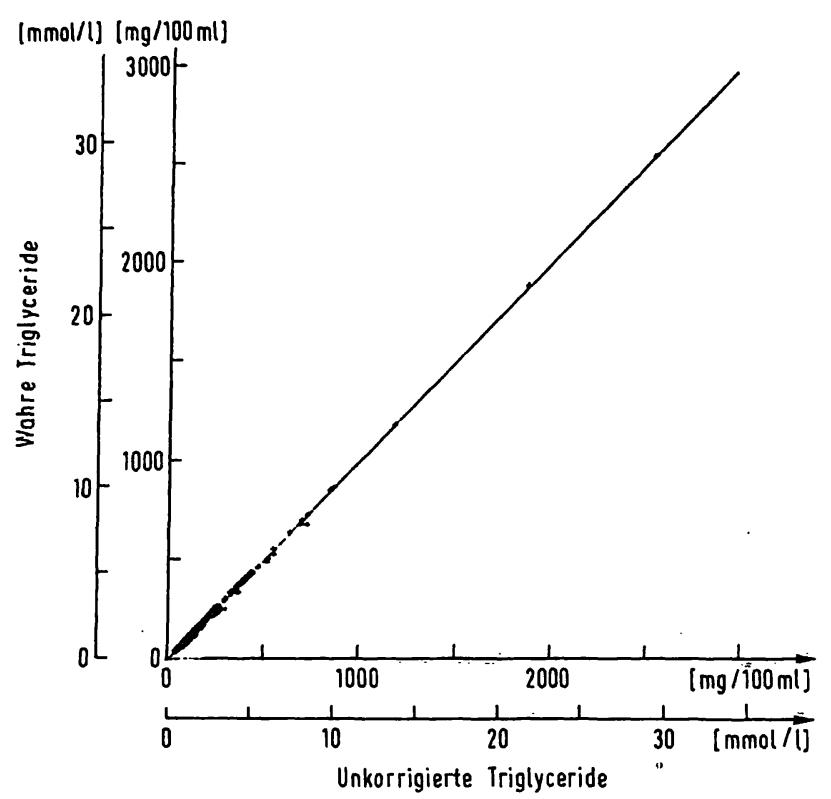

Abb. 6. Korrclation unkorrigierter Triglyceride $z u$ wahren Triglyceriden

$\mathrm{N}=142$

$\mathrm{y}=\mathrm{a}+\mathrm{bx}$

$a=-0,097$ (für $\mathrm{mmol} / \mathrm{l}) \mathrm{a}=-8,5($ für $\mathrm{mg} / 100 \mathrm{ml}$ )

$\mathrm{b}=0.955 \pm 0,002$

$r=0,999 \pm 0.002$

$\overline{\mathrm{x}}=2,91 \pm 3,40$ (für $\mathrm{mmol} / \mathrm{l}) \overline{\mathrm{x}}=258 \pm 301$ (für $\mathrm{mg} / 100 \mathrm{ml}$ )

$\bar{y}=2,80 \pm 3,38($ für mmol/l) $\bar{y}=248 \pm 300($ für mg/ $100 \mathrm{ml}$ )
Der hohe Korrelationskoeffizient von 0,999 ist damit zu erklären, daß die Konzentration des freien Glycerins im Verhältnis zum Triglycerid-Glycerin nur gering ist und somit keinen wesentlichen Einfluß auf den TriglyceridWert ausübt. Aus einer Steigung von 0,995 und einem Schnittpunkt mit der $y$-Achse bei - 0,097 ist abzulesen, daß die ,wahren“"Triglycerid-Werte im Mittel um einen systematischen Betrag unter den „unkorrigierten“"Triglycerid-Werten liegen. Im Bereich 1-23 mmol/1 (89-2036 mg/100 ml) Triglyceride, berechnet aus dem Gesamt-Glycerin-Gehalt, liegt der wahre Triglycerid-Wert um 0,114-0,228 mmol/1 (10-20 mg Triglyceride/100 ml) Serum niedriger. Es kann also die bisherige Praxis, pauschal $0,114 \mathrm{mmol} / 1$ ( $10 \mathrm{mg}$ Triglyceride $/ 100 \mathrm{ml}$ ) Serum als Anteil des freien Glycerins vom Gesamt-Glycerin-Gehalt (unkorrigierter Triglycerid-Wert) abzuziehen, beibehalten werden.

\section{Danksagung}

Wir danken den med.-techn. Assistentinnen Frau H. Dietrich und Frl. $D$. Bauder für ihre exakte und fleißige Mitarbeit.

\section{Literaturverzeichnis}

1. Barbosiak, J. J., Rimm, A. A., Anderson, A. J., Tristani, F. E. Walker, J. A. \& Flemma, R. J. (1974). Amer. Heart J. 87, 716-721.

2. Eggstein, M. \& Kreutz, F. H. (1966). Klin. Wochenschr. 44, 262-267.

3. Empfehlungen der Deutschen Gesellschaft für Klinische Chemic zur Durchführung klinisch-chemischer Untersuchungen bei der Prüfung von Arzneimitteln (1976). diese Z. 14, 161-164.

4. Soloni, F. G. (1971). Clin. Chem. 17, 529-534.

5. Tiffany, T. O., Morten, J. M., Hall, E. M. \& Garrett, A. S., Jr. (1974). Clin. Chem. 20, 476-481.

6. Giegel, J. L., Ham, Anna B. \& Clema, W. (1975). Clin. Chem. $21,1575-1581$.

7. Neri, B. P. \& Frings, C. S. (1973). Clin. Chem. 19, 1201-1202.

8. Köhring, B. \& Kattermann, R. (1974). diese Z. 12, 282-286.
9. Bucolo, G. \& David. H. (1973). Clin. Chem. 19, 476-482.

10. Wahlefeld, A. W. (1974) in Methoden der enzymatischen Analyse (Bergmeyer, H. U. ed.), 3. Aufl., S. 1878-1882. Verlag Chemie, Weinheim.

11. Wahlefeld, A., Klose, S. \& Munz, E. (1975). IX. Int. Congr. of Clinical Chemistry, Toronto, Canada, 13.-18. Juli. Abstracts, S. 25.

12. Liebich, H. M., Müller, P. H., Schmülling, R.-M., Eggstein, M., Stähler, F. \& Weisshaar, D. (1977). diese Z. 15, 503-508.

13. Eggstein, M. (1965). Klin. Wochenschr. 43, 1031-1038.

14. Eggstein, M. (1961). Habilitationsschrift, Marburg/Lahn.

15. Eggstein, M. \& Kuhlmann, Elisabeth (1974) in Methoden der enzymatischen Analyse (Bergmeyer, H. U. ed.), 3. Aufl., S. 1871-1878. Verlag Chemie, Weinheim.

16. Schmülling, R.-M., Liebich, H., Locher, M., Mildner, I. \& Eggstein, M. (1973). diese Z. 11, 513-520.
Dipl.-Biochem. Dr. med. Peter H. Müller Medizinische Univ.-Klinik Abteilung Innere Medizin IV Auf dem Schnarienberg D-7400 Tübingen 